\title{
A New Golden Age of Minimal Surfaces
}

\author{
Joaquín Pérez
}

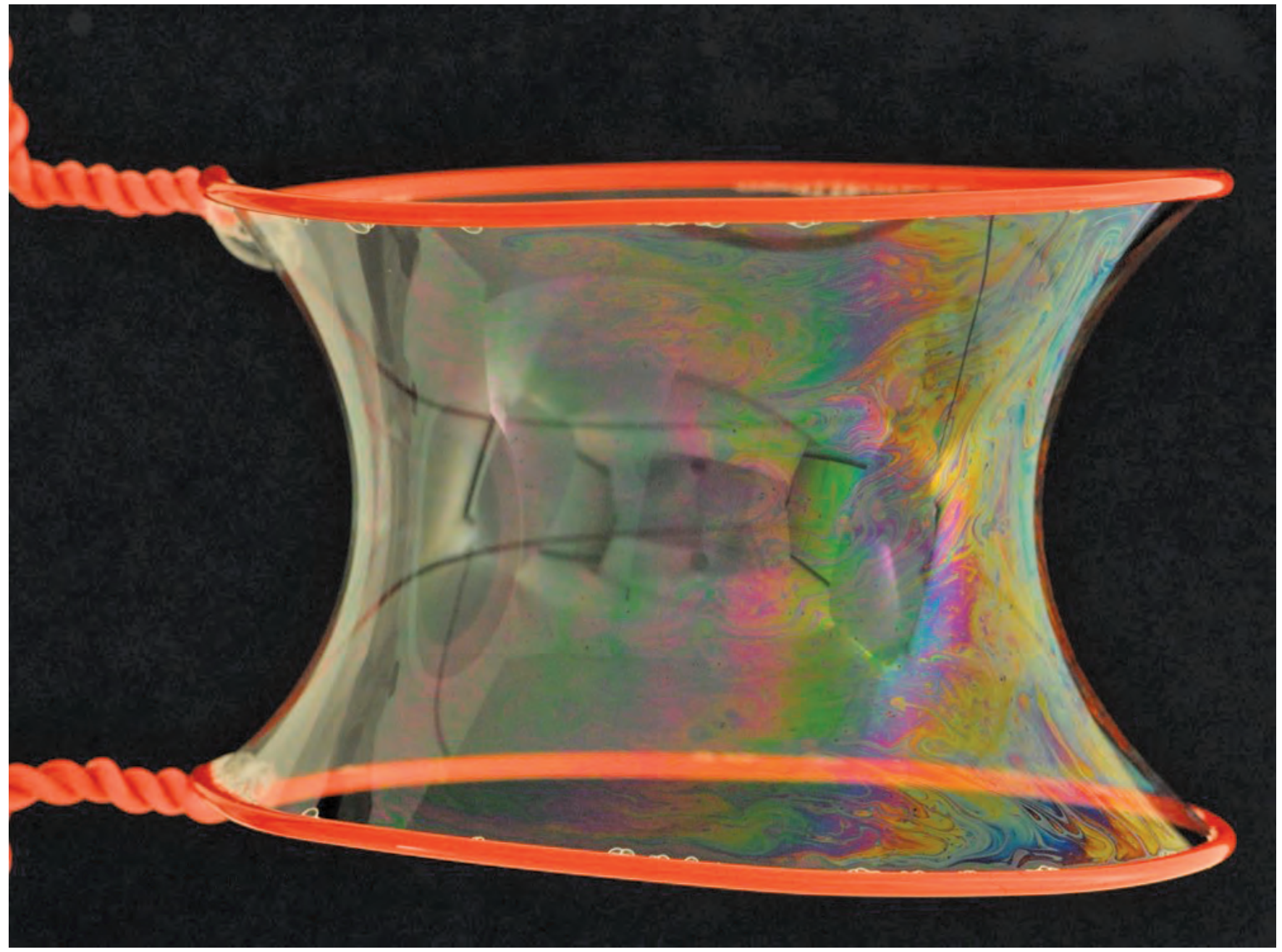

Figure 1. A minimal surface, like this soap film, is characterized by the property that small pieces minimize area for given boundary.

Joaquín Pérez is professor of geometry and topology at the University of Granada and director of the Research Mathematics Institute IEMath-GR. His e-mail address is jperez@ugr.es.

Research partially supported by the Spanish MEC/FEDER grant No. MTM2014-52368-P.

For permission to reprint this article, please contact:

reprint-permission@ams.org.

DOI: http://dx.doi.org/10.1090/noti1500
Abstract. We give a brief tour of some of the recent developments in classical minimal surface theory, especially those where the work of Colding and Minicozzi on compactness properties of embedded minimal disks in Euclidean three-space has been instrumental. Along the way, we will discuss some of the main open problems. This article is a translated and revised version of an expository paper to appear in the Gaceta de la Real Sociedad Matemática Española. 


\section{Introduction}

A minimal surface, like the soap film of Figure 1, has the property that small pieces minimize area for a given boundary, even though the whole surface may be unstable. At first, there were few explicit examples (see Figure 2): the plane, the catenoid of Euler (1741), and the helicoid of Meusnier (1776).

Many of the greatest mathematicians in history have been challenged by minimal surfaces; some of them made spectacular advances in a relatively definite period, producing golden ages of this theory: The first one occurred approximately in the period 1830-1890, when renowned mathematicians such as Enneper, Scherk, Schwarz, Riemann, and Weierstrass made major advances on minimal surfaces through the application of the newly created field of complex analysis by providing analytic formulas for a general minimal surface. Also in this period, fundamental research by Plateau on surface tension gave a physical interpretation to the problem of minimizing area with a given contour, which allowed the spread of this minimization problem beyond mathematics, to the point that since then it is customary to refer to it as the Plateau problem. A second golden age of minimal surfaces took place from about 1914 to 1950, with the incipient theory of partial differential equations: here, we highlight the contributions of Bernstein, Courant, Douglas (who in 1936 won the first Fields Medal ${ }^{1}$ for his solution of the Plateau problem), Morrey, Morse, Radó, and Shiffman. A third golden age started in the 1960s, when giants of the stature of Almgren, Alt, Calabi, do Carmo, Chern, Federer, Finn, Fleming, Gackstatter, Gulliver, Hardt, Hildebrandt, Jenkins, Lawson, Nitsche, Osserman, Serrin, Simon, and Simons opened new routes through the use of multiple
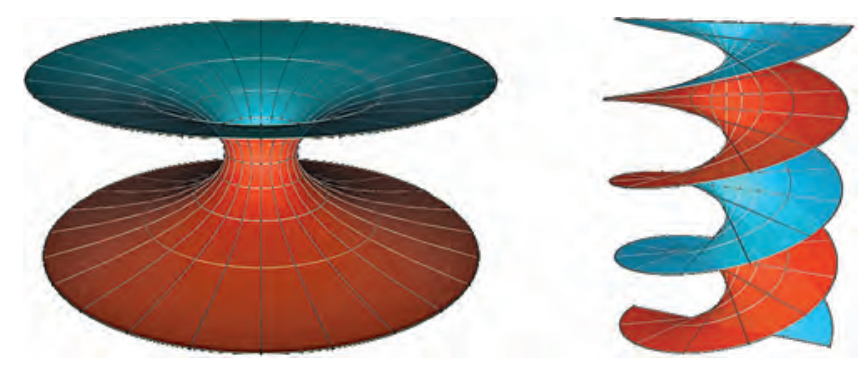

Figure 2. The first explicit examples of minimal surfaces were the plane, the catenoid (Euler, 1741), and the helicoid (Meusnier, 1776).

techniques, from Riemann surfaces to geometric measure theory, passing through integrable systems, conformal geometry, and functional analysis. The appearance of computers was crucial for the discovery in the eighties of new examples of complete minimal surfaces without selfintersections. The abundance of these newly discovered examples led to new problems and conjectures about the classification and structure of families of surfaces with prescribed topology. More recent major contributors are too numerous to list here.

\footnotetext{
${ }^{1}$ Shared with Ahlfors for his work on Riemann surfaces.
}

In this article we hope to convince the reader that as with the previous milestones, we are currently witnessing a new golden age of minimal surfaces, mostly favored by a new tool discovered in 2004: the so-called ColdingMinicozzi theory. This work, published in an impressive series of four articles in the same issue of Annals of Mathematics [2], analyzes the convergence of sequences of embedded minimal disks without imposing a priori uniform bounds on area or curvature. We will sketch how this theory has helped to solve open problems that were considered inaccessible until recently, and we will venture, with all the reservations that predictions deserve, to expose some of the most interesting open problems in this field.

\section{The theory of minimal surfaces is a confluence of many branches of mathematics.}

In order to develop these objectives in a limited number of pages, we must pay the price of not going into detail. There are many articles, books, and chapters of books where interested readers can satisfy their curiosity, such as the volume by Colding and Minicozzi [1] or the survey by Meeks and me [3].

\section{Basic Results}

The theory of minimal surfaces is a confluence of many branches of mathematics. We can define minimality in at least eight different but equivalent ways, based on the theory that we are most passionate about.

Let $X: M \rightarrow \mathbb{R}^{3}$ be an isometric immersion of a Riemannian surface in three-dimensional Euclidean space, and let $N: M \rightarrow \mathbb{S}^{2}(1) \subset \mathbb{R}^{3}$ be its unit normal or Gauss map (here $\mathbb{S}^{2}(1)$ denotes the sphere of radius 1 and center the origin of $\mathbb{R}^{3}$ ). If we perturb $X$ in a relatively compact domain $\Omega \subset M$ by a compactly supported differentiable function $f \in C_{0}^{\infty}(\Omega)$, then $X+t f N$ is again an immersion for $|t|<\varepsilon$ and $\varepsilon>0$ small enough. The mean curvature $H \in C^{\infty}(M)$ of $X$ (arithmetic mean of the principal curvatures) is related to the area functional $A(t)=\operatorname{Area}((X+t f N)(\Omega))$ by means of the first variation of area formula:

$$
A^{\prime}(0)=-2 \int_{\Omega} f H d A
$$

where $d A$ is the area element of $M$. Now we can state the first two equivalent definitions of minimality.

Definition 1. A surface $M \subset \mathbb{R}^{3}$ is minimal if it is a critical point of the area functional for all variations with compact support.

Definition 2. A surface $M \subset \mathbb{R}^{3}$ is minimal when its mean curvature vanishes identically.

Locally and after a rotation, every surface $M \subset \mathbb{R}^{3}$ can be written as the graph of a differentiable function $u=u(x, y)$. In 1762, Lagrange wrote the foundations of the calculus of variations by finding the PDE associated 
to a critical point of the area functional when the surface is a graph:

Definition 3. A surface $M \subset \mathbb{R}^{3}$ is minimal if around any point it can be written as the graph of a function $u=u(x, y)$ that satisfies the second-order, quasi-linear elliptic partial differential equation

$$
\left(1+u_{x}^{2}\right) u_{y y}-2 u_{x} u_{y} u_{x y}+\left(1+u_{y}^{2}\right) u_{x x}=0 .
$$

The above PDE can also be written in divergence form:

$$
\operatorname{div}\left(\frac{\nabla u}{\sqrt{1+|\nabla u|^{2}}}\right)=0 .
$$

Neglecting the gradient in the denominator of (3) leads to the celebrated Laplace equation. This means that on a small scale (where $u$ is close to a constant), minimal surfaces inherit properties of harmonic functions, such as the maximum principle, Harnack's inequality, and others. On a large scale, dramatic changes appear in the way that global solutions to the Laplace and minimal surface equations behave; perhaps the paradigmatic example of this dichotomy is Bernstein's theorem: the only solutions of (3) defined in the whole of $\mathbb{R}^{2}$ are the affine functions, while of course there are many global harmonic functions.

A consequence of the second variation of area formula (i.e., the expression for $\left.A^{\prime \prime}(0)\right)$ shows that every minimal surface minimizes area locally. This property justifies the word minimal for these surfaces (not to be confused with being a global area minimizer, which is a much more restrictive property: the unique complete surfaces in $\mathbb{R}^{3}$ that minimize area globally are the affine planes).

Definition 4. A surface $M \subset \mathbb{R}^{3}$ is minimal if every point $p \in M$ admits a neighborhood that minimizes area among all surfaces with the same boundary.

Definitions 1 and 4 place minimal surfaces as 2dimensional analogues of geodesics in Riemannian geometry and connect them with the calculus of variations. Another functional of great importance is the energy,

$$
E=\int_{\Omega}|\nabla X|^{2} d A,
$$

where again $X: M \rightarrow \mathbb{R}^{3}$ is an isometric immersion and $\Omega \subset M$ is a relatively compact domain. Area and energy are related by the inequality $E \geq 2 A$, with equality occurring exactly when $X$ is conformal. The fact that every Riemannian surface admits local conformal (isothermal) coordinates allows us to give two other equivalent definitions of minimality.

Definition 5. A conformal immersion $X: M \rightarrow \mathbb{R}^{3}$ is minimal if it is a critical point of the energy functional for every compactly supported variation or, equivalently, when every point on the surface admits a neighborhood that minimizes energy among all surfaces with the same boundary.

The classical formula $\Delta X=2 H N$ that links the Laplacian of an isometric immersion $X: M \rightarrow \mathbb{R}^{3}$ with its mean curvature function $H$ and Gauss map $N$ leads us to the next definition.
Definition 6. An isometric immersion $X=\left(x_{1}, x_{2}, x_{3}\right)$ : $M \rightarrow \mathbb{R}^{3}$ of a Riemannian surface in three-dimensional Euclidean space is said to be minimal if its coordinate functions are harmonic: $\Delta x_{i}=0, i=1,2,3$.

From a physical point of view, the so-called Young's equation shows that the mean curvature of a surface separating two media expresses the difference of pressures between the media. When both media are under the same pressure, the surface that separates them is minimal. This happens after dipping a wire frame (mathematically, a nonnecessarily planar Jordan curve) in soapy water. However, soap bubbles that we all have blown have nonzero constant mean curvature, because they enclose a volume of air whose pressure is greater than the atmospheric pressure.

Definition 7. A surface $M \subset \mathbb{R}^{3}$ is minimal if each point $p \in M$ has a neighborhood that matches the soap film spanned by the boundary of this neighborhood.

To give the last definition of minimality, remember that the differential $d N_{p}$ at each point $p \in M$ of the Gauss map $N$ is a self-adjoint endomorphism of the tangent plane $T_{p} M$. Therefore, there exists an orthonormal basis of $T_{p} M$ where $d N_{p}$ diagonalizes (principal directions at $p$ ), being the opposite of the eigenvalues of $d N_{p}$, the so-called principal curvatures of $M$ at $p$. As the mean curvature $H$ is the arithmetic mean of the principal curvatures, the minimality of $M$ is equivalent to the vanishing of the trace of $d N_{p}$ or, equivalently, to the property that the matrix of $d N_{p}$ in any orthonormal basis of $T_{p} M$ is of the form

$$
d N_{p}=\left(\begin{array}{cc}
a & b \\
b & -a
\end{array}\right) .
$$

After identifying $N$ with its stereographic projection onto the extended complex plane, the Cauchy-Riemann equations allow us to enunciate the eighth equivalent version of minimality.

Definition 8. A surface $M \subset \mathbb{R}^{3}$ is minimal when its stereographically projected Gauss map $g: M \rightarrow \mathbb{C} \cup\{\infty\}$ is a meromorphic function.

In fact, for a minimal surface $M \subset \mathbb{R}^{3}$, not only is the Gauss map meromorphic but also the whole immersion can be expressed by means of holomor-
Minimal surfaces appear frequently in nature. phic data: as the third coordinate function $x_{3}$ of $M$ is a harmonic function, then it admits locally a conjugate harmonic function $x_{3}^{*}$. Thus, the height differential $d h:=d x_{3}+i d x_{3}^{*}$ is a welldefined holomorphic 1-form on $M$, and the surface can be conformally parameterized by the explicit formula (4)

$$
X: M \rightarrow \mathbb{R}^{3}, X(p)=\mathfrak{R} \int_{p_{0}}^{p}\left(\frac{1}{2}\left(\frac{1}{g}-g\right), \frac{i}{2}\left(\frac{1}{g}+g\right), 1\right) d h,
$$

where $\mathfrak{R}$ stands for real part and $p_{0}$ is the point of $M$ that we choose to be sent by $X$ to the origin in $\mathbb{R}^{3}$ (i.e., 

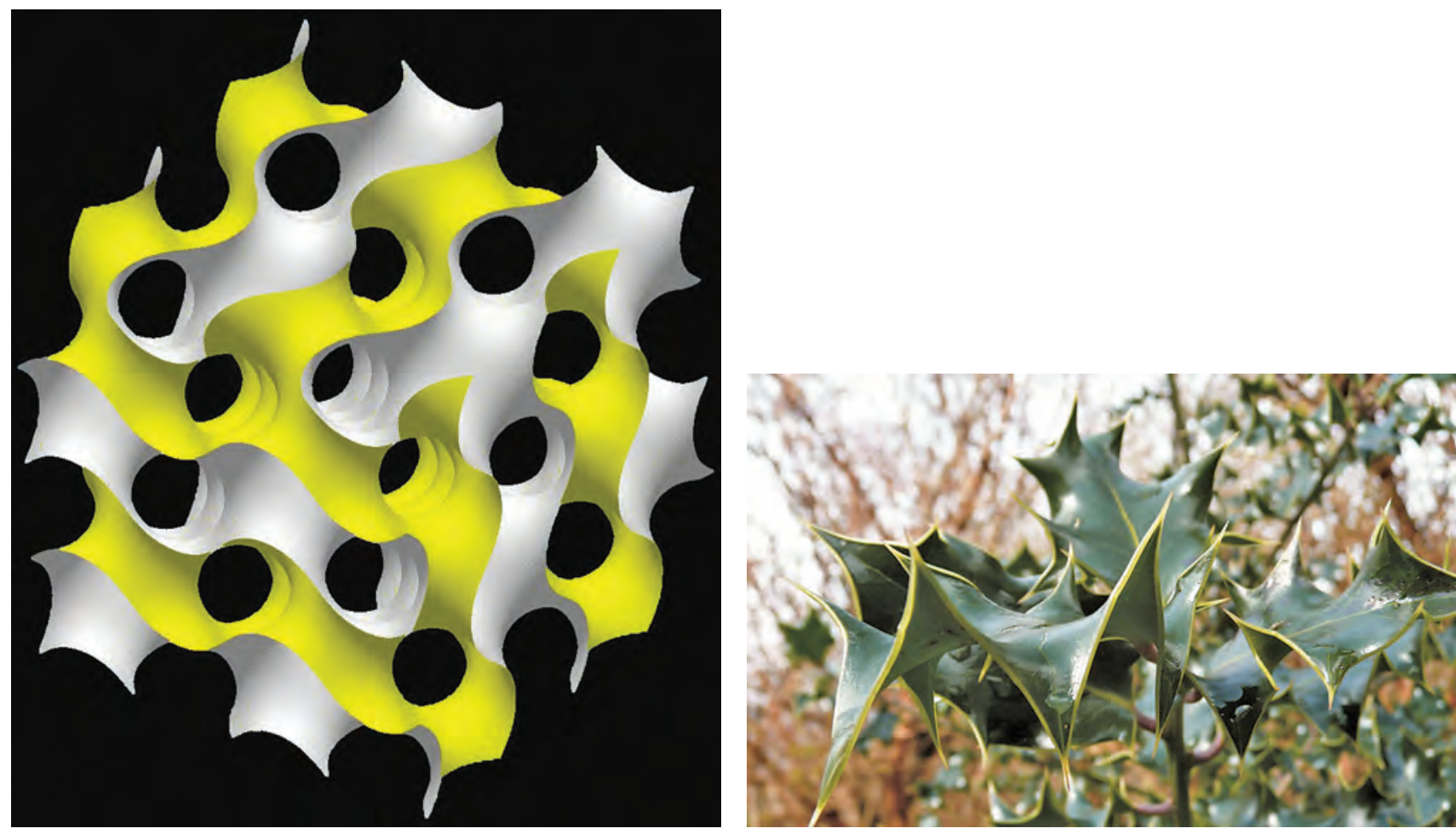

Figure 3. (left) The gyroid (A. Schoen, 1970) has been observed in diblock copolymer systems; (right) nature often seeks optimal forms in terms of perimeter and area such as minimal surfaces.

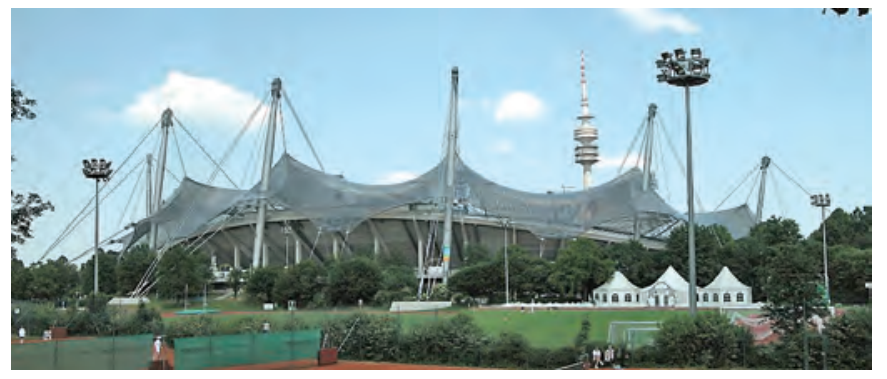

Figure 4. Frei Otto modeled the Olympic stadium in Munich on minimal surfaces.

formula (4) defines $X$ up to an ambient translation). The pair $(g, d h)$ is usually called the Weierstrass data of $M$.

Minimal surfaces appear frequently in nature, not only in soap films or, more generally, interfaces separating immiscible fluids at the same pressure but also for example in diblock copolymers, smectic liquid crystals (materials that have uniformly spaced layers with fluidlike order within each layer), crystallography, semiconductor technology,...even in the cuticular structure in the wing scales of certain insects! See Figure 3.

Minimization properties for this class of surfaces have motivated renowned architects such as Frei Otto to use them to design optimal structures such as the cover of the Olympic stadium in Munich (Figure 4). The beauty of their balanced forms has awakened the interest of sculptors such as Robert Engman and Robert Longhurst. From a purely mathematical viewpoint, minimal surfaces have been studied in other ambient spaces besides Euclidean space, giving rise to applications in such diverse problems as the positive mass and the Penrose conjectures in mathematical physics, the Smith conjecture on diffeomorphisms of finite order of the three-dimensional sphere, and Thurston's geometrization conjecture in 3-manifold theory.

\section{Classical Minimal Surface Theory}

By classical theory we will mean the study of connected, orientable, complete, embedded minimal surfaces in $\mathbb{R}^{3}$. Let $\mathcal{M}_{C}$ be the class of complete embedded minimal surfaces $M \subset \mathbb{R}^{3}$ with finite genus. In order to understand this last word, recall that the maximum principle for harmonic functions implies that there are no compact minimal surfaces without boundary in $\mathbb{R}^{3}$; therefore, complete minimal surfaces must have topological ends (roughly speaking, ways to go to infinity intrinsically on the surface). After compactifying topologically a minimal surface $M$ by adding a point to each end, we define the genus of $M$ as the genus of its compactification. If $g \in \mathbb{N} \cup\{0\} \cup\{\infty\}$ and $k \in \mathbb{N} \cup\{\infty\}$, we let $\mathcal{M}_{C}(g, k)$ be the subset of $\mathcal{M}_{C}$ that consists of those surfaces with genus $g$ and $k$ topological ends. When both $g$ and $k$ are finite, we will say that the surface has finite topology.

A surface $M \subset \mathbb{R}^{3}$ is called proper if every intrinsically divergent sequence of points of $M$ also diverges in $\mathbb{R}^{3}$. Roughly speaking, a complete surface is proper when its topological ends are placed at infinity in $\mathbb{R}^{3}$ (an infinite 
roll of paper that wraps infinitely often and limits to an infinite cylinder from its inside or outside is an example of a complete surface that is not proper). We will denote by $\mathcal{M}_{P}$ the subset of $\mathcal{M}_{C}$ formed by the proper minimal surfaces, and we let $\mathcal{M}_{P}(g, k)=\mathcal{M}_{P} \cap \mathcal{M}_{C}(g, k)$.

Our goal in this section is to describe the main examples of minimal surfaces in these families, attending to their topology, conformal structure, asymptotic behavior, and the main results of classification. As we go through this description, we will discuss some of the most interesting open problems.

\section{Complete Minimal Surfaces with Finite Topology}

The trivial example in this class is the plane. The first nontrivial examples of minimal surfaces (Figure 2) belong to this class: the catenoid discovered by Euler in 1741 (genus zero and two ends) and the helicoid found by Meusnier in 1776 (genus zero, one end). Both surfaces support multiple characterizations; among the most classic ones we will mention that the catenoid is the unique minimal surface of revolution together with the plane (Euler) and that the helicoid and the plane are the unique ruled minimal surfaces (Catalan). A special mention among examples in this family is deserved by the Costa torus, the first complete minimal surface of finite topology discovered after the aforementioned ones (after 206 years!), which has genus 1 and 3 ends, and its generalization to any finite genus $g \geq 1$, discovered by Hoffman and Meeks, also with three ends; see Figure 5.

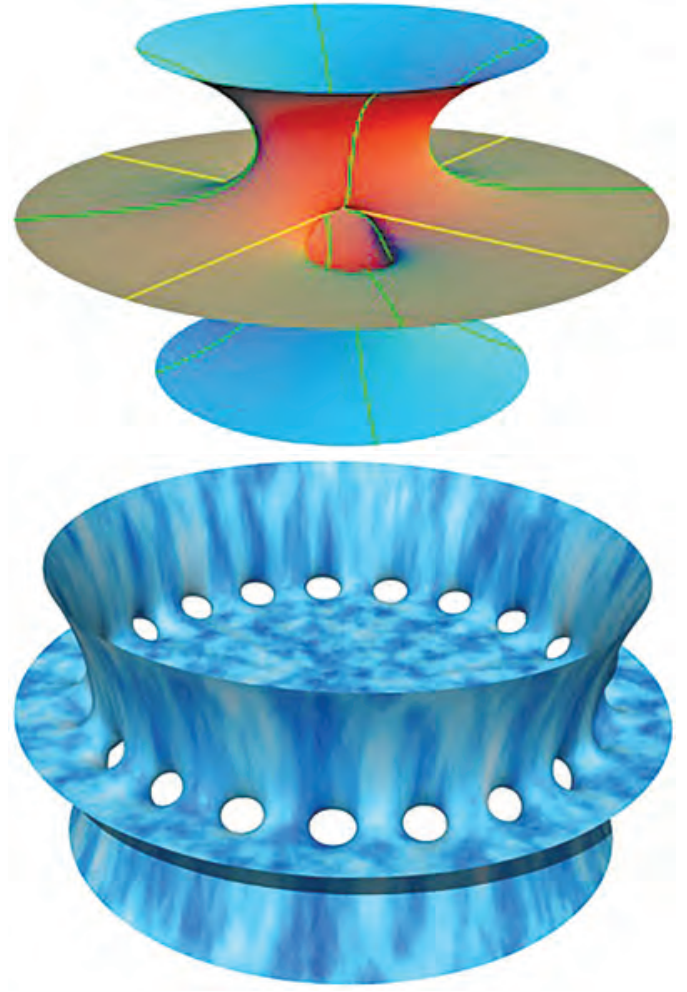

Figure 5. (top) The Costa torus; (bottom) a HoffmanMeeks minimal surface.
Regarding the relationship between $\mathcal{M}_{C}$ and $\mathcal{M}_{P}$ in the case of finite topology, we should highlight a deep result of Colding and Minicozzi which asserts that every complete embedded minimal surface with finite topology is proper. Its proof is an application of the famous theory by Colding and Minicozzi, a topic which we will talk about a little later.

For our discussion of the case of finite topology, we will distinguish two subcases, depending on whether the number of ends of the minimal surface is one or more than one.

Surfaces with finite genus and one end. In 2005, Meeks and Rosenberg applied Colding-Minicozzi theory to show that the plane and the helicoid are the only possible examples in $\mathcal{M}_{P}(0,1)$ (i.e., they gave the full classification of the simply connected properly embedded minimal surfaces). By the above properness result of Colding and Minicozzi, the same uniqueness result holds in $\mathcal{M}_{C}(0,1)$. As for the asymptotic behavior of surfaces in $\mathcal{M}_{P}(g, 1)=\mathcal{M}_{C}(g, 1)$ with $1 \leq g<\infty$, Bernstein and Breiner proved in 2011 that every surface in $\mathcal{M}_{P}(g, 1)$ is asymptotic to a helicoid and conformally parabolic. ${ }^{2}$ For this reason, surfaces in $\mathcal{M}_{P}(g, 1)$ are usually called helicoids of genus $g$. On existence results in this line, it is worth mentioning that Hoffman, Weber, and Wolf discovered in 2009 a helicoid of genus one with the conformal structure of a rhombic torus minus one point and that Hoffman, Traizet, and White have recently proven the existence of examples in $\mathcal{M}_{P}(g, 1)$ for each finite $g \geq 1$ (arXiv 2015). An important open problem about $\mathcal{M}_{P}(g, 1)$ is the possible uniqueness of examples with a given genus: this uniqueness is known in the case $g=0$, and it is conjectured that there exists a unique helicoid of genus $g$ for each $g \geq 1$, but even the local version of this result is not known.

Surfaces with finite genus and $k$ ends, $2 \leq k<\infty$. The main structural result in this case is due to Collin, who proved in 1997 that if $M \in \mathcal{M}_{P}(g, k)$ has $g, k$ finite and $k \geq 2$, then $M$ lies in a particularly well-studied family: surfaces with finite total curvature, i.e., those where the Gaussian curvature $K$ is integrable:

$$
\int_{M} K d A=-\int_{M}|K| d A>-\infty
$$

(Note that since the mean curvature, the sum of the principal curvatures, is zero, the Gauss curvature, the product of the principal curvatures, is nonpositive.) By previous work of Huber and Osserman, condition (5) implies that $M$ is conformally equivalent to a compact Riemann surface $\mathbb{M}$ of genus $g$ to which we have removed $k$ points (in particular, $M$ is conformally parabolic), and both the Gauss map $g: M \rightarrow \mathbb{C} \cup\{\infty\}$ and the height differential $d h$ of $M$ extend to holomorphic objects defined on $\mathbb{M}$. This allows the application of powerful tools of complex analysis and algebraic geometry of compact Riemann surfaces; in some way, and given the lack of compactness of a complete minimal surface in $\mathbb{R}^{3}$, those with finite total curvature are the closest ones to being compact.

${ }^{2} M$ is conformally parabolic if it does not admit any nonconstant, nonpositive subharmonic function. 
The asymptotic behavior of these minimal surfaces is also well known: every end is asymptotic to a plane or half-catenoid. On uniqueness results, we highlight the following ones:

1. Schoen proved in 1983 that if $M \in \mathcal{M}_{C}(g, 2)$ has finite total curvature, then $M$ is a catenoid. This is an application of the famous reflection method of moving planes of Alexandrov, which is based on the maximum principle for the equation (2).

2. In 1991, López and Ros characterized the catenoid as the only surface in $\mathcal{M}_{C}(0, k)$ with finite total curvature together with the plane. Again the idea is based on the maximum principle, but now applied to what has since been dubbed the López-Ros deformation, a 1-parameter family of minimal surfaces defined in terms of the Weierstrass data $(g, d h)$ of a given minimal surface $M$. The López-Ros deformation only exists under a certain hypothesis on the flux map of the original surface. ${ }^{3}$

3. In 1984, Costa classified the surfaces in $\mathcal{M}_{C}(1,3)$ with finite total curvature. These surfaces reduce to the Costa torus and a 1-parametric family of thrice-punctured tori discovered by Hoffman and Meeks by deforming the Costa torus (and studied later by Hoffman and Karcher).

The previous result by Costa was the first complete description of a moduli space $\mathcal{M}_{C}(g, k)$ that does not reduce to a single surface: $\mathcal{M}_{C}(1,3)$ has the structure of a noncompact 1-dimensional manifold, identifiable with an open interval. Generalizing this result, in 1996 Pérez and Ros endowed the moduli spaces $\mathcal{M}_{C}(g, k)(0 \leq g<\infty$, $2 \leq k<\infty)$ with a differentiable structure of dimension $k-2$ around each minimal surface $M \in \mathcal{M}_{C}(g, k)$ with an additional nondegeneracy assumption that affects the linear space of Jacobi functions on $M$, which are the solutions $u: M \rightarrow \mathbb{R}$ to the second-order, linear elliptic PDE

$$
\Delta u-2 K u=0 \quad \text { on } M,
$$

where $K$ is the Gaussian curvature of $M$. Until now, all known examples in $\mathcal{M}_{C}(g, k)$ satisfy this nondegeneracy hypothesis. We highlight that the dimension of the space of nondegenerate surfaces in $\mathcal{M}_{C}(g, k)$ does not depend on the genus $g$, but only on the number of ends $k$.

A major open problem is the Hoffman-Meeks conjecture: If $M \in \mathcal{M}_{C}(g, k)$, then $k \leq g+2$. The best known result to date in this regard is due to Meeks, Pérez, and Ros (arXiv 2016), who proved the existence of an upper bound for $k$ depending only on $g$, again by application of the Colding-Minicozzi theory.

Another important open problem consists of deciding if there exist surfaces in some moduli space $\mathcal{M}_{C}(g, k)$ that do not satisfy the nondegeneracy condition mentioned above, and if they do exist, provide any "reasonable"

\footnotetext{
${ }^{3}$ The flux of a minimal surface $M \subset \mathbb{R}^{3}$ is the linear map $F: H_{1}(M) \rightarrow \mathbb{R}^{3}$ that associates to each 1-dimensional homology class $[c] \in H_{1}(M)$ the integral along a representative $c \in[c]$ of the unit vector field along $c$ that is tangent to $M$ and orthogonal to c. The condition for the López-Ros deformation to be well defined on $M$ is that the range of $F$ is at most 1.
}

structure to the space $\mathcal{M}_{C}(g, k)$ around such a singular surface (as an orbifold?).

\section{Minimal Surfaces with Infinite Topology}

Next we enter the world of classical minimal surfaces with infinite topology, i.e., those that have either infinitely many ends or infinite genus. The most basic examples in this family were discovered by Riemann in the nineteenth century (and posthumously published by his disciple Hattendorf) and consist of a 1-parametric family of properly embedded minimal surfaces, invariant by a translation, with genus zero and infinitely many ends asymptotic to equally spaced parallel planes. The Riemann minimal examples admit the following fascinating characterization: together with the plane, the helicoid and the catenoid, they are the unique properly embedded minimal surfaces in $\mathbb{R}^{3}$ that can be foliated by circles and lines in parallel planes (indeed, Riemann discovered these examples by imposing this property); see Figure 6.

The Riemann minimal examples show how the periodicity of a surface can be regarded as a method to produce examples of infinite topology: if the quotient surface by the group of isometries is not simply connected, then the lifted surface in $\mathbb{R}^{3}$ has infinite topology. The same thing happens with other examples of minimal surfaces discovered in the nineteenth century, such as those shown in Figure 6:

1. The singly periodic Scherk minimal surface (second from the left in Figure 6) is invariant by a cyclic group of translations. The quotient surface by the cyclic group has genus zero and four ends (asymptotic to half-planes; these ends are called Scherk type ends); viewed in $\mathbb{R}^{3}$, this surface has infinite genus and one end. We can see this example as a desingularization of two orthogonal planes by introducing infinitely many alternating holes forming a $45^{\circ}$ angle with the planes along their line of intersection. As in the case of the Riemann minimal examples, the singly periodic Scherk minimal surface can be deformed by a 1-parametric family of singly periodic, properly embedded minimal surfaces, obtained by desingularization of planes that intersect with an angle $\theta \in(0, \pi)$.

2. The doubly periodic Scherk minimal surface (third from the left in Figure 6) is invariant by an infinite group generated by two translations of linearly independent vectors. Again, the quotient surface has genus zero and four ends (of Scherk type); viewed in $\mathbb{R}^{3}$, this surface has infinite genus and one end. It can be considered as the desingularization of two infinite families of equally spaced vertical halfplanes, one family inside $\{(x, y, z) \mid z>0\}$ and the other one in $\{(x, y, z) \mid z<0\}$, in such a way that half planes in different families cut at right angles. This surface also lies in a 1-parameter family of properly embedded, doubly periodic minimal surfaces, each of which desingularizes two infinite families of vertical half-planes in the open upper and lower half-spaces of $\mathbb{R}^{3}$, where the parameter is the angle 

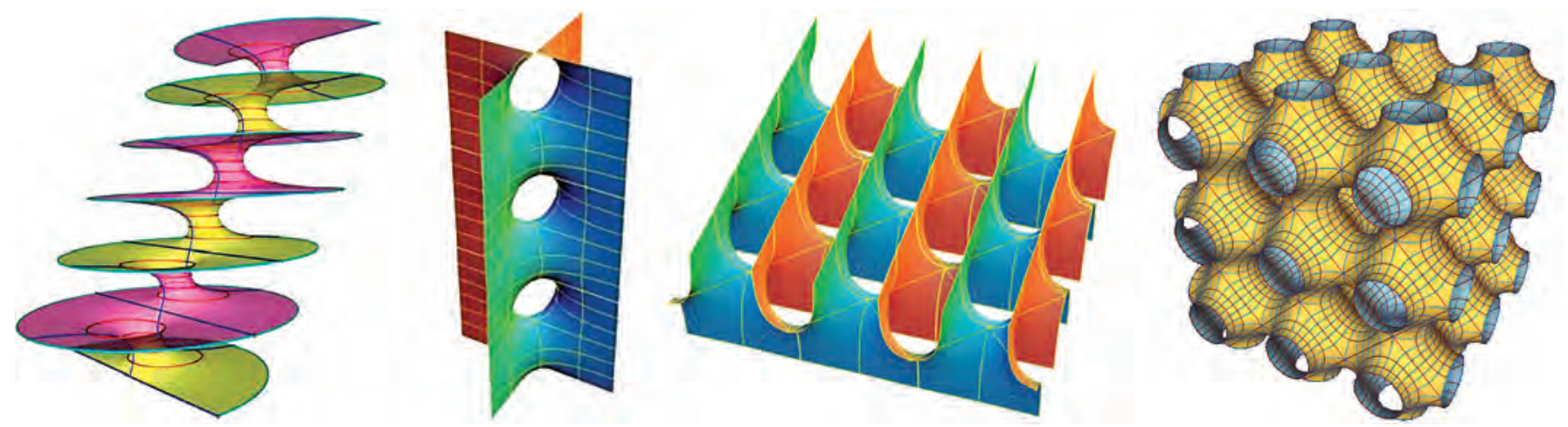

Figure 6. From left to right: a Riemann minimal example, singly and doubly periodic Scherk surfaces, and the triply periodic Schwarz $P$-surface.

$\theta \in(0, \pi)$ that the half-planes in the two families form. There is a direct relationship between the Scherk singly and doubly minimal surfaces, which reflects the fact that every harmonic function admits (locally) a conjugate harmonic function.

3. The triply periodic Schwarz $P$-surface (Figure 6, right) is invariant by the group generated by three translations of linearly independent vectors. The quotient surface by this lattice of translations is compact with genus three and lives in a threedimensional cubic torus. Viewed in $\mathbb{R}^{3}$, this surface has infinite genus and one end. The Schwarz Psurface is one of the most famous triply periodic minimal surfaces, a class of surfaces with multiple applications to crystallography and material science: the isometry group of each triply periodic minimal surface $M \subset \mathbb{R}^{3}$ is a crystallographic group, and the quotient surface $M$ over the lattice $\Gamma$ of translations of rank 3 that leaves $M$ invariant divides the three-dimensional torus $\mathbb{R}^{3} / \Gamma$ into two regions of equal volume called labyrinths. The gyroid (Figure 3, left) is another famous triply periodic minimal surface with compact quotient of genus three. The classification of triply periodic embedded minimal surfaces with quotient of genus three (the lowest possible nontrivial value) is another major open problem.

In view of the above examples, we could ask ourselves if the only method of producing minimal surfaces with infinite topology is by imposing periodicity. The answer is no, as shown in 2007 by Hauswirth and Pacard, who used gluing techniques ${ }^{4}$ to merge a Hoffman-Meeks minimal surface (we mentioned these surfaces when describing examples in $\mathcal{M}_{C}(g, 3)$ in the subsection "Complete Minimal Surfaces with Finite Topology") with two halves of a Riemann minimal surface $\mathcal{R}$. In Figure 7 (left) we can see a schematic representation of one of the examples by Hauswirth and Pacard, when the central surface to be

\footnotetext{
${ }^{4}$ This technique consists of a sophisticated application of the implicit function theorem to the mean curvature operator defined between certain Banach spaces.
}

merged is the Costa torus (i.e., $g=1$ ). Also by gluing techniques, but using Riemann surfaces with nodes, Traizet was able to prove in 2012 the existence of a complete, nonperiodic minimal surface with infinite genus and infinitely many ends asymptotic to half-catenoids (Figure 7 right). In summary, there are lots of examples in the case of infinite topology.

As for uniqueness results for minimal surfaces of infinite topology, it is clear in light of the previous paragraph that we must distinguish in some way the families that we have found: a reasonable starting point could be imposing some kind of periodicity. Here, it is worth mentioning the following classification results for moduli spaces of periodic minimal surfaces with prescribed topology:

1. The Riemann minimal examples are the unique properly embedded minimal tori with finitely many planar ends in a quotient of $\mathbb{R}^{3}$ by a translation (Meeks, Pérez, and Ros, 1998). The number of ends must be even, and when we fix this number the corresponding moduli space is a noncompact manifold of dimension 1 .

2. The Scherk doubly periodic minimal surfaces are the unique properly embedded minimal surfaces with genus zero and finitely many ends in a quotient of $\mathbb{R}^{3}$ by two independent translations (Lazard-Holly and Meeks, 2001). Again the number of ends is necessarily even, and for a fixed number of ends, the moduli space is diffeomorphic to an open interval.

3. The moduli spaces of properly embedded minimal tori with any fixed finite number of parallel planar ends in a quotient of $\mathbb{R}^{3}$ by two linearly independent translations were described in 2005 by Pérez, Rodríguez, and Traizet. Each of these moduli spaces (for any fixed even number of ends) is a noncompact manifold of dimension 3 whose surfaces are called KMR examples (in honor of Karcher, Meeks, and Rosenberg, who previously found 1-parameter families of these surfaces in this moduli space).

4. The moduli spaces of properly embedded minimal surfaces with genus zero and finitely many ends of Scherk type in a quotient of $\mathbb{R}^{3}$ by a translation were 

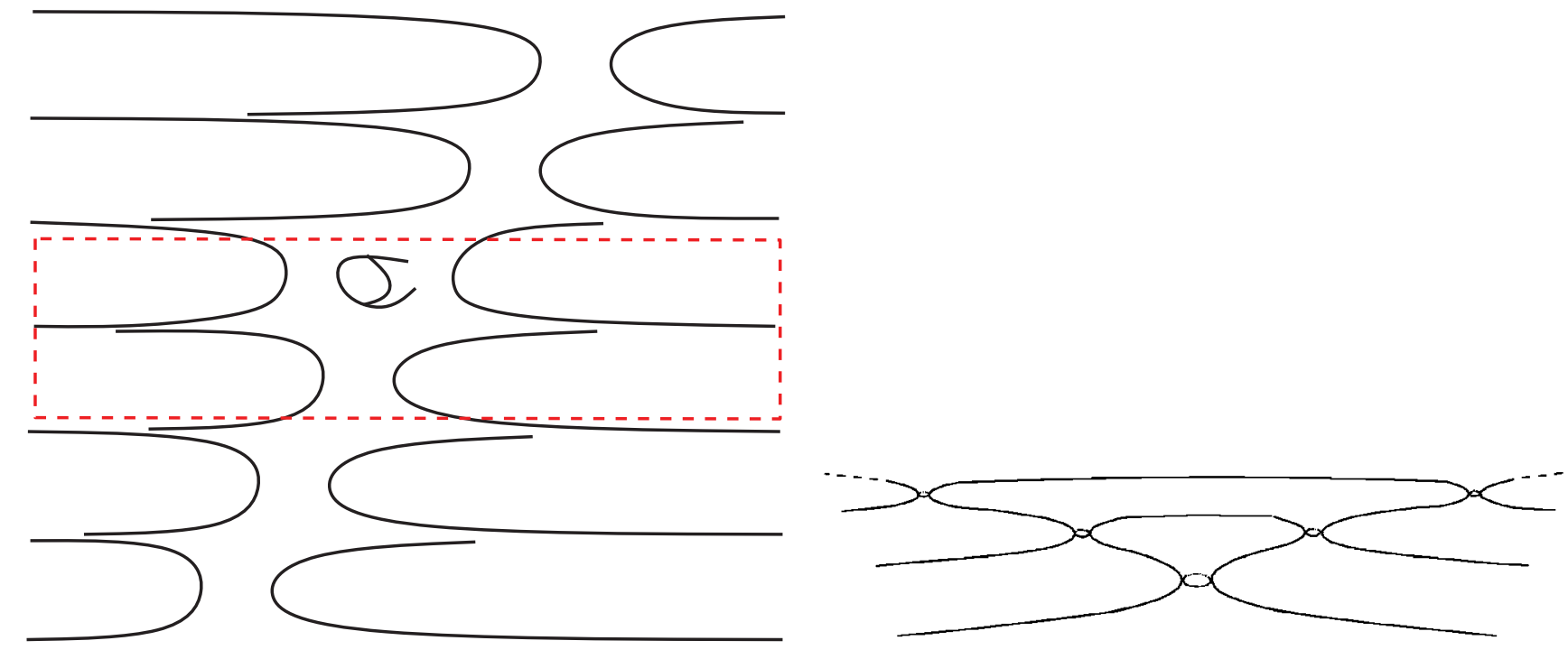

Figure 7. From left to right: schematic representations of a Hauswirth-Pacard minimal surface with genus 1 and of the example of infinite genus by Traizet: two nonperiodic, complete embedded minimal surfaces with infinite topology.

classified in 2007 by Pérez and Traizet. In this case, these moduli spaces are noncompact manifolds of dimension $2 k-3$ (here $2 k$ is the number of ends), whose surfaces were discovered by Karcher in 1988 as a generalization of the Scherk simply periodic minimal surfaces.

The four uniqueness results listed above have a common flavor. First, periodicity is used in a strong way, since it allows working in the quotient of $\mathbb{R}^{3}$ by the corresponding group of isometries, and the quotient minimal surfaces always have finite total curvature in the sense of equation (5); in this setting, one can control the asymptotic geometry and the conformal representation of the minimal surfaces under study. Second, the desired uniqueness follows from a continuity argument: one starts by proving that any surface $M$ in each of these moduli spaces can be deformed within the moduli space (openness part) until arriving at a point $M_{\infty}$ in the boundary of the moduli space, which turns out to be a properly embedded minimal surface with simpler topology or periodicity than those in the original moduli space (compactness part); this compactness requires a rather complete understanding of the possible limits of sequences of minimal surfaces in the original moduli space. Once we have arrived at $M_{\infty}$, the desired global uniqueness follows from an inverse function theorem argument (local uniqueness around $M_{\infty}$ ) that needs the previous classification of the moduli space of minimal surfaces to which $M_{\infty}$ belongs. This last aspect reveals a stratified structure in the moduli spaces of embedded minimal surfaces with prescribed topology and periodicity: the boundary of a given moduli space is the union of other moduli spaces of minimal surfaces with simpler topology or periodicity. For instance, the description of the moduli space in item 3 above requires solving the classification problem in item 4.
The strategy sketched in the preceding paragraph fails badly if we seek classification results for minimal surfaces with infinite topology without imposing periodicity, but in this case the Colding-Minicozzi theory is of great help, as we will explain next.

\section{Colding-Minicozzi Theory}

Consider the following question:

Problem 9. What are the properly embedded minimal surfaces in $\mathbb{R}^{3}$ with genus zero?

Suppose that $M \subset \mathbb{R}^{3}$ is a surface that meets the conditions of Problem 9. As explained above, in the case that $M$ has only one end we know that $M$ is a plane or a helicoid (Meeks and Rosenberg). If $M$ has $k$ ends with $2 \leq k<\infty$, then $M$ is a catenoid by the theorems of Collin and López-Ros. It remains to study the case that $M$ has infinitely many ends. If we knew that such an $M$ were invariant by a translation $T$, then it would not be difficult to check that the quotient surface of $M$ by the cyclic group generated by $T$ is a torus with finitely many ends, and hence $M$ is a Riemann minimal example by the 1998 result of Meeks, Pérez, and Ros. Therefore, a way of solving Problem 9 is to prove that if the number of ends of $M$ is infinite, then $M$ is periodic.

Often we face the problem of understanding the possible limits of a sequence of embedded minimal surfaces. As a trivial example, let us think of a surface $M \subset \mathbb{R}^{3}$ that is invariant by a translation of vector $v \in \mathbb{R}^{3}-\{0\}$. The constant sequence $\left\{M_{n}:=M-n v=M\right\}_{n \in \mathbb{N}}$ has as trivial limit $M$ itself. This naive example suggests a possible way to solve Problem 9: suppose that a properly embedded minimal surface $M \subset \mathbb{R}^{3}$ with infinitely many ends is a solution to this problem. As the number of ends of $M$ is infinite, one can deduce that $M$ has infinite total curvature, whence we can find a divergent sequence of points $p_{n} \in M$ 
where the unit normal to $M$ takes the same value. It is reasonable to try to conclude that $\left\{M_{n}=M-p_{n}\right\}_{n}$ has (at least) a convergent subsequence as a step to demonstrate the desired periodicity of $M$. We have thus transformed Problem 9 into another one, perhaps more ambitious:

Problem 10. Under what conditions can we extract a convergent subsequence from a given sequence of embedded minimal surfaces?

Suppose that $\left\{M_{n}\right\}_{n}$ is a sequence of embedded minimal surfaces in an open set $A \subset \mathbb{R}^{3}$. Also assume that $\left\{M_{n}\right\}_{n}$ has at least one accumulation point, since we do not want the $M_{n}$ to completely escape and have nothing to analyze in the limit. Each surface $M_{n}$ can be locally written as the graph of a function $u_{n}$ defined in an open subset of the tangent plane of $M_{n}$ at a given point, and the size of the domain of $u_{n}$ can be uniformly controlled if we have uniform local bounds for the Gaussian curvatures (equivalently, for the second fundamental forms) of the surfaces. If in addition we have uniform local area bounds for the $M_{n}$, then we will control the number of graphs that lie in a given region of $A$. Therefore, working in a very small (but uniform) scale, we will deduce that every surface $M_{n}$ gives rise to a single graphing function $u_{n}$. Thus we have transformed Problem 10 about convergence of surfaces into another problem, the convergence of graphs. In this setting, the uniform local curvature bounds for the $M_{n}$ produce equicontinuity of the $u_{n}$, and the fact that we are working locally produces uniform boundedness for the $u_{n}$. Therefore, the Arzelá-Ascoli Theorem insures that a subsequence of the $u_{n}$ converges uniformly to a limit function $u_{\infty}$ that can be proven to satisfy the same PDE (2) as the $u_{n}$. A prolongation argument now implies that a subsequence of $\left\{M_{n}\right\}_{n}$ converges to an embedded minimal surface in $A$, and thus our Problem 10 is solved in this case.

If we do not have local area bounds for the $M_{n}$ but still assume local uniform curvature bounds, reasoning similar to the above leads to the conclusion that a subsequence of $\left\{M_{n}\right\}_{n}$ converges to a natural generalization of the notion of minimal surface: a lamination whose leaves are minimal surfaces. Without going into detail, a lamination $\mathcal{L}$ of $A$ is a closed union (in the induced topology on $A$ ) of surfaces embedded in $A$, called leaves of $\mathcal{L}$, with a certain local product structure. This means that we can take local coordinates in $A$ that transform the leaves into the product of a two-dimensional disk with a closed subset of $\mathbb{R}$, which we can think of as the heights of disjoint copies of that disk placed horizontally (see Figure 8). This local product structure endows the leaves of $\mathcal{L}$ with the structure of smooth, pairwise disjoint surfaces. A lamination is said to be minimal if its leaves are all minimal surfaces. For example, if $Z$ is a nonempty closed subset of $\mathbb{R}$, then the collection of horizontal planes $\mathcal{L}_{Z}=\left\{P_{Z}=\mathbb{R}^{2} \times\{z\} \mid Z \in Z\right\}$ is a minimal lamination of $A=\mathbb{R}^{3}$ whose leaves are the planes $P_{Z}$. In the case that a lamination $\mathcal{L}$ of $A$ does not leave any empty spaces in $A$, we call it a foliation of $A\left(\mathcal{L}_{Z}\right.$ is a foliation of $\mathbb{R}^{3}$ when $\left.Z=\mathbb{R}\right)$. The theory of minimal laminations is a natural extension of the one of minimal surfaces. However, we still have not

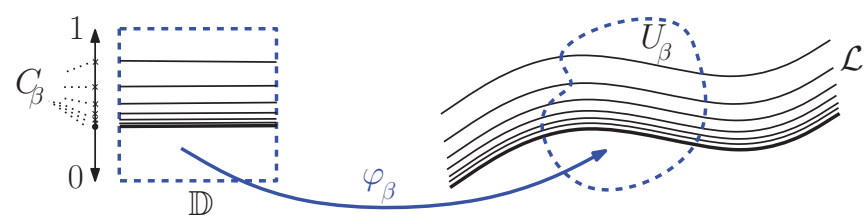

Figure 8 . The open set $A$ is covered by images $U_{\beta}$ of local charts $\varphi_{\beta}$, each one transforming a collection of disks at heights lying in a closed subset $C_{\beta}$ of $[0,1]$ into portions of the leaves of $\mathcal{L}$.

provided any examples of a nontrivial minimal lamination of $\mathbb{R}^{3}$ that does not consist of a single embedded minimal surface other than a collection of planes $\mathcal{L}_{Z}$ as above.

Coming back to our Problem 10, what can we say about the limit of the $M_{n}$ if these embedded minimal surfaces do not have uniform local bounds for their second fundamental forms? Here is where the theory of Colding-Minicozzi comes to our aid. Following the previous notation, the lack of uniform local curvature bounds implies that the Gaussian curvature of the $M_{n}$ blows up at some point of $A$; i.e., the following set is nonempty:

$$
\widehat{S}=\left\{x \in A|\sup | K_{M_{n} \cap \overline{\mathbb{B}}(x, r)} \mid \rightarrow \infty, \forall r>0\right\},
$$

where $K_{\Sigma}$ denotes the Gaussian curvature of a surface $\Sigma$ and $\overline{\mathbb{B}}(x, r)$ is the closed ball centered at $x \in \mathbb{R}^{3}$ with radius $r>0$. The Colding-Minicozzi theory describes the limit of (a subsequence of) the $M_{n}$ in the above scenario under an additional hypothesis: each $M_{n}$ must be topologically a compact disk that is contained in a ball of radius $R_{n}>0$, say centered at the origin, with boundary $\partial M_{n}$ contained in the boundary sphere of that ball. The description of this limit is very different depending upon whether the sequence of radii $R_{n}$ diverges or stays bounded.

Theorem 11 (Colding-Minicozzi). Given $n \in \mathbb{N}$, let $M_{n}$ be an embedded minimal disk in a closed ball $\overline{\mathbb{B}}\left(R_{n}\right)=$ $\overline{\mathbb{B}}\left(\overrightarrow{0}, R_{n}\right)$ with $\partial M_{n} \subset \partial \mathbb{B}\left(R_{n}\right)$. If $R_{n} \rightarrow \infty$ and $\hat{S} \cap \mathbb{B}(1) \neq \varnothing$, then a subsequence of the $M_{n}$ converges to a foliation of $\mathbb{R}^{3}$ by parallel planes, away from a straight line ${ }^{5}$ (called the singular set of convergence), along which the curvature of $M_{n}$ blows up when $n \rightarrow \infty$.

To better understand the last result, we will use the following example. Consider the standard vertical helicoid $H=\{(x, y, z) \mid x \sin z=y \cos z\}$. Take a sequence of positive numbers $\lambda_{n}$ tending to zero, and consider for each $n \in \mathbb{N}$ the homothetic copy $M_{n}=\lambda_{n} H$ of $H$ by ratio $\lambda_{n}$ that is again minimal and simply connected. As $n$ increases, $M_{n}$ can be thought of as a new view of $H$ from a viewpoint that becomes further and further away, as in Figure 9. The farther away we look at the helicoid $H$, the more it looks like a collection of horizontal planes separated by smaller and smaller distances, and so in the

\footnotetext{
${ }^{5}$ In fact, Colding and Minicozzi proved that the singular set of convergence is a Lipschitz arc transverse to the limit foliation. Using the uniqueness of the helicoid as the only nonflat surface in $\mathcal{M}_{P}(0,1)$, Meeks deduced in 2004 that this Lipschitz arc is indeed a line.
} 


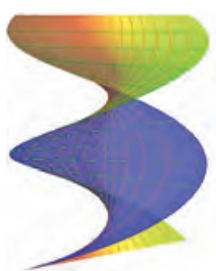

$H$
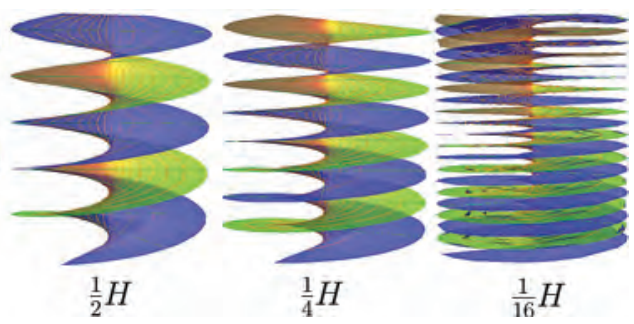

Figure 9. Homothetic images of the same vertical helicoid $H$, with ratio $\lambda_{n}=1 / 2^{n}$.

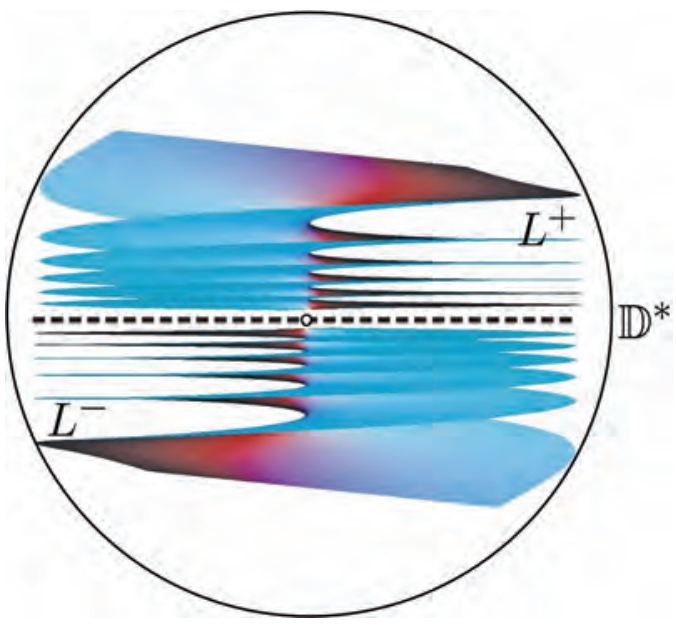

Figure 10. A minimal lamination of the punctured unit ball, with three leaves and a singularity at the origin.

limit we obtain the foliation of $\mathbb{R}^{3}$ by horizontal planes. Observe that each leaf of this limit foliation is flat (its Gaussian curvature is identically zero) and the Gaussian curvatures of the $M_{n}$ converge to zero away from the $Z$-axis. However, since the Gaussian curvature of $H$ along the $z$-axis is constant -1 , the Gaussian curvature of $M_{n}$ along the same axis is $-1 / \lambda_{n}^{2}$, which tends to infinity. In other words, the singular set $\hat{S}$ defined in (6) is the $z$-axis in this example. Also note that the limit foliation is perfectly regular along $\hat{S}$; it is only the convergence of the $M_{n}$ to the limit that fails along $\hat{S}$. This limit object is known as a limiting parking garage structure with one column: away from the $z$-axis, the structure becomes arbitrarily flat and horizontal (this is where cars park), and to travel from one parking floor to another one, cars have to go up the ramp (around the column at the $z$-axis). Well, Theorem 11 tells us that the general behavior of the limit of the embedded minimal disks $M_{n}$ when the radii $R_{n}$ tend to infinity is essentially the same as this example.

The description when the radii $R_{n}$ remain bounded can also be visualized with an example. In 2003, Colding and Minicozzi produced a sequence of minimal disks $M_{n}$ embedded in the closed unit ball $\bar{B}(1)$ and of helicoidal appearance, such that the number of turns that the boundary curve $\partial M_{n}$ makes around the $z$-axis tends to infinity as $n \rightarrow \infty$, and the limit of the $M_{n}$ is a minimal lamination $\mathcal{L}$ of $\mathbb{B}(1)-\{\overrightarrow{0}\}$ that consists of three leaves: one is the horizontal punctured unit disk
$\mathbb{D}^{*}=\left\{(x, y, 0) \mid 0<x^{2}+y^{2}<1\right\}$, and the other two are nonproper minimal surfaces $L^{+}, L^{-}$that rotate infinitely many times from above and below $\mathbb{D}^{*}$, accumulating on $\mathbb{D}^{*}$ as in Figure 10. In this case, the Gaussian curvature of the $M_{n}$ blows up at the origin, but this time $\overrightarrow{0}$ is a genuine singularity of the limit lamination $\mathcal{L}$, which does not admit a smooth extension across $\overrightarrow{0}$.

The theoretical description by Colding and Minicozzi for the limit of a sequence of compact, embedded minimal disks $M_{n} \subset \overline{\mathbb{B}}\left(R_{n}\right)$ with $\partial M_{n} \subset \partial \mathbb{B}\left(R_{n}\right)$ and bounded radii $R_{n}$ is very technical, and we will omit it here. Instead, we will simply mention that after extracting a subsequence, the $M_{n}$ converge to a minimal lamination with singularities. The singularities of such a limit singular minimal lamination form a closed set, and each singularity is of one of the following two types:

(a) Isolated singularities, in which case Figure 10 shows essentially the behavior of the limit object: there is a leaf $D^{*}$ of the lamination that limits to the singularity $p$ (in fact, $D^{*}$ extends smoothly across $p$ to an embedded minimal disk $D$ ) and one or two nonproper leaves, which rotate infinitely many times and accumulate at $D^{*}$. Furthermore, portions of the $M_{n}$ outside a solid cone of axis $p+\left(T_{p} D\right)^{\perp}$ can be written as multivalued graphs over annular regions of $T_{p} D$, and as $n \rightarrow \infty$, these annular regions converge to a punctured disk, at the same time that the number of turns of the multivalued graphs inside the $M_{n}$ become arbitrarily large and the multivalued graphs collapse into $D^{*}$ as in Figure 11 (left).

(b) Nonisolated singularities, each of which is the limit of at least one sequence of isolated singularities, as in Figure 11 (right).

It should also be noted that in the above description, the set $\hat{S}$ where the Gaussian curvatures of the disks $M_{n}$ blow up consists of not only the singularities of the limit lamination $\mathcal{L}$ but also possibly embedded arcs of class $C^{1,1}$ around which $\mathcal{L}$ is a local foliation, as in Figure 11 (left). In particular, these arcs are not singularities of $\mathcal{L}$ except for their end points, as in the convergence of the $M_{n}$ to $\mathcal{L}$ in Theorem 11 .

The previous description leads us directly to the study of the singularities of a minimal lamination of an open subset of $\mathbb{R}^{3}$. Does this set have any reasonable structure? This question is another open central problem in minimal surface theory. Along this line, it is worth mentioning two recent results of Meeks, Pérez, and Ros (2016): 

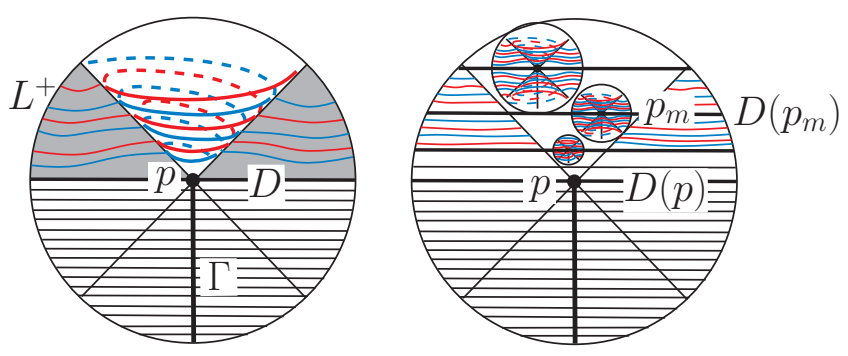

Figure 11. Left: schematic representation of an isolated singularity $p$ of a singular minimal lamination $\mathcal{L}$ obtained as a limit of embedded minimal disks $M_{n}$, with a nonproper leaf $L^{+}$at one side of the disk leaf $D$ that passes through $p$. Locally around $p$ and outside a solid cone of vertex $p$ and axis $p+\left(T_{p} D\right)^{\perp}, L^{+}$is a multivalued graph. The other side of $D$ is foliated by leaves of $\mathcal{L}$. The convergence is singular along a $C^{1,1}$ arc $\Gamma$. Right: at a nonisolated singularity $p$, the disk leaf $D=D(p)$ passing through $p$ is also the limit of the corresponding disk leaves $D\left(p_{m}\right)$ associated to isolated singularities $p_{m}$ that converge to $p$.

Theorem 12 (Local Removable Singularity Theorem). Let $\mathcal{L} \subset \mathbb{B}(1)-\{\overrightarrow{0}\}$ be a minimal lamination. Then $\mathcal{L}$ extends to a minimal lamination of $\mathbb{B}(1)$ (i.e., the singularity at $\overrightarrow{0}$ is removable) if and only if the Gaussian curvature function $K_{\mathcal{L}}$ of the lamination does not blow up at the origin faster than the square of the extrinsic distance to $\overrightarrow{0}$; i.e., $\left|K_{\mathcal{L}}\right|(x) \cdot\|x\|^{2}$ is bounded in $\mathcal{L}$.

It follows from Theorem 12 that if the function $\left|K_{\mathcal{L}}\right|(x)$. $\|x\|^{2}$ is bounded in a minimal lamination $\mathcal{L} \subset \mathbb{B}(1)-\{\overrightarrow{0}\}$, then $\left|K_{\mathcal{L}}\right|(x) \cdot\|x\|^{2}$ extends across the origin with value zero. Another consequence of this theorem is that in the example of Figure 10, the Gaussian curvature of the disks $M_{n}$ blows up faster than the square of the distance to the origin as $n \rightarrow \infty$.

Another result about singularities of minimal laminations is a global version of Theorem 12 that classifies the minimal laminations of $\mathbb{R}^{3}-\{\overrightarrow{0}\}$ with quadratic decay of curvature:

Theorem 13. Let $\mathcal{L} \subset \mathbb{R}^{3}-\{\overrightarrow{0}\}$ be a nonflat minimal lamination such that $|K|(x) \cdot\|x\|^{2}$ is bounded. Then $\mathcal{L}$ extends across the origin to a minimal lamination of $\mathbb{R}^{3}$ that consists of a single leaf $M$, which is a properly embedded minimal surface with finite total curvature. In particular, $|K|$ decays much faster than quadratically with the distance to the origin: $|K|(x) \cdot\|x\|^{4}$ is bounded in $M$.

We have said that in order to apply the theory of Colding-Minicozzi to a sequence $M_{n}$ of embedded minimal surfaces, we need to assume that the $M_{n}$ are compact disks with boundaries in ambient spheres. This condition is not really a restriction, as it can be naturally obtained by a rescaling argument so that the injectivity radius function of the rescaled minimal surfaces is uniformly bounded away from zero (Meeks, Pérez, and Ros [4]).

\section{Classification of the Properly Embedded Minimal Surfaces in $\mathbb{R}^{3}$ with Genus Zero}

To finish our brief tour through the current state of the classical minimal surface theory, we return to Problem 9 on the properly embedded minimal surfaces $M$ in $\mathbb{R}^{3}$ with genus zero. In the first paragraph of the section "ColdingMinicozzi Theory" we explained that the problem reduces to proving that if $M$ has infinitely many ends, then $M$ is periodic. This strategy, which uses Colding-Minicozzi theory as we have mentioned above, was the one used by Meeks, Pérez, and Ros [5] to prove the following result:

Theorem 14. Every properly embedded minimal surface $M \subset \mathbb{R}^{3}$ with genus zero is either a plane, a helicoid, a catenoid, or one of the Riemann minimal examples. In particular, $M$ is foliated by circles or straight lines in parallel planes.

A final remark about the proof of Theorem 14 is in order. The theory of Colding and Minicozzi yields only the quasi-periodicity of $M$ (this means that if $\left\{p_{n}\right\}_{n}$ is a divergent sequence of points in $M$, then a subsequence of $\left\{M-p_{n}\right\}_{n}$ converges to a properly embedded minimal surface in $\mathbb{R}^{3}$ with genus zero and infinitely many ends). The key to proving the desired periodicity of $M$ once we know it is quasi-periodic is a fascinating application of the theory of integrable systems and more precisely of the holomorphic Korteweg-de Vries equation, a third-order PDE that models mathematically the behavior of waves on shallow water surfaces.

\section{References}

[1] T. H. Colding and W. P. Minicozzi II, Minimal Surfaces, volume 4 of Courant Lecture Notes in Mathematics, New York University, Courant Institute of Mathematical Sciences, New York, 1999. MR1683966, Zbl 0987.49025.

[2] _ , The space of embedded minimal surfaces of fixed genus in a 3-manifold, Parts I-IV, Ann. of Math. (2) 160 (2004). MR2119718, MR2123932, MR2123933.

[3] W. H. MEeKs III and J. PÉREZ, The classical theory of minimal surfaces, Bulletin of the AMS, (N.S.) 48 (2011), 325-407. MR2801776, Zbl 1232.53003.

[4] W. H. MeEKS III, J. PÉREZ, and A. Ros, The local picture theorem on the scale of topology, to appear in J. Differential Geometry, preprint at https://arxiv.org/abs/1505.06761.

[5] __ Properly embedded minimal planar domains, Ann. of Math. (2) 181 (2015), 473-546 MR3275845, Zbl 06399442. 

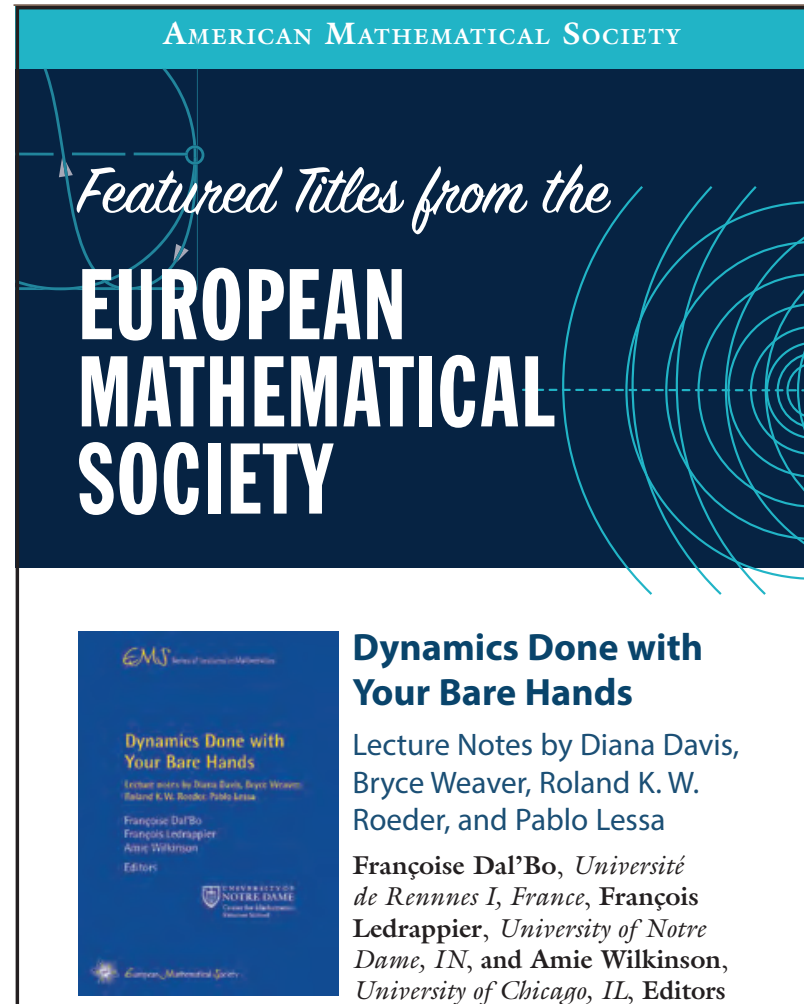

\section{Dynamics Done with Your Bare Hands}

Lecture Notes by Diana Davis, Bryce Weaver, Roland K. W. Roeder, and Pablo Lessa

Françoise Dal'Bo, Université de Rennnes I, France, François Ledrappier, University of Notre Dame, $I N$, and Amie Wilkinson, University of Chicago, IL, Editors

Based on four lectures given at the Undergraduate Summer School of the Thematic Program Dynamics and Boundaries, this volume introduces (under)graduate students to the field of dynamical systems by emphasizing elementary examples, exercises, and bare hands constructions.

EMS Series of Lectures in Mathematics, Volume 26;2016; 214 pages; Softcover; ISBN: 978-3-037|9-I68-2; List US\$44;AMS members US $\$ 35.20$; Order code EMSSERLEC/26

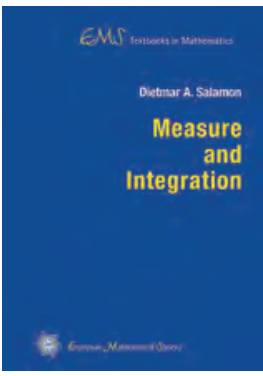

\section{Measure and} Integration

Dietmar A. Salamon, ETH

Zurich, Switzerland

Assuming familiarity with first-year analysis and linear algebra, this book is intended as a companion to a one-semester introductory lecture course on measure and integration.

EMS Textbooks in Mathematics, Volume 18; 2016; 363 pages; Hardcover; ISBN: 978-3-037|9-|59-0; List US\$58; AMS members US\$46.40; Order code EMSTEXT//8

Publications of the European Mathematical Society (EMS). Distributed within the Americas by the American Mathematical Society.

View the complete collection of titles that we have to offer at bookstore.ams.org

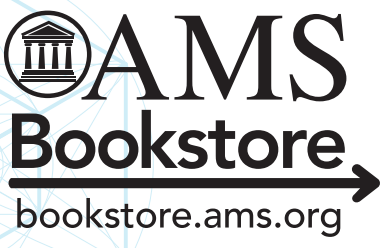

\section{Credits}

Figure 1 image taken from soapbubble.dk.

Figure 2 is courtesy of Matthias Weber.

Figure 3 image taken from hyperbolic-crochet.blogspot.com . es /2012_07_01_archive.htm7; courtesy of Daina Taimina.

Figure 4 is courtesy of Fritz-Geller-Grimm.

Figures 5 and 6 are courtesy of Matthias Weber.

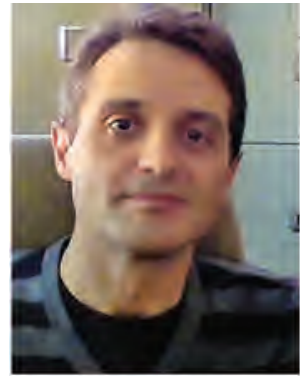

Joaquín Pérez

\section{ABOUT THE AUTHOR}

Joaquín Pérez received a $\mathrm{PhD}$ in mathematics from the University of Granada under Antonio Ros in 1996. He is a member of the International Relations Committee of the Royal Mathematical Society of Spain and director of the Research Mathematical Institute IEMath-GR, one of the four venues of the Spanish Institute of Mathematics. 